J. Clin. Chem. Clin. Biochem.

Vol. 15,1977 , pp. $663-668$

\title{
Neue Ergebnisse der Zuckeranalytik von Liquor cerebrospinalis
}

\author{
Von R. Seuffer \\ Hygiene-Institut (Direktor: Prof. Dr. R.-E. Bader) der Universität Tübingen
}

W. Voelter und H. Bauer

Institut für Organische Chemie der Universität Tübingen

(Eingegangen 25. Juli 1975/2. Juni 1977)

Zusammenfassung: Es wird über den flüssigkeitschromatographischen Nachweis von Fructose, Galaktose, Glucose, Mannose, Rhamnose und Ribose im Liquor cerebrospinalis von 24 Patienten berichtet. Die hohe Empfindlichkeit (Nachweisgrenze: 0,05 bis $0,23 \mu \mathrm{g}$ pro Komponente), Auflösung und Spezifität der hier beschriebenen Analysenmethode erlaubt es, Kohlenhydrate in Körperflüssigkeiten ohne Anreicherung zu bestimmen. Das Verfahren eröffnet neue Möglichkeiten in der medizinischen Diagnostik.

\section{A new method for the determination of sugars in cerebrospinal fluid}

Summary: The determination of fructose, galactose, glucose, mannose, rhamnose and ribose, using a newly developed liquid chromatography sugar analyzer is reported. High sensitivity (detection limit: 0.05 to $0.23 \mu \mathrm{g}$ per compound), resolution and specifity of the method permits the routine detection of carbohydrates in biological fluids without prior extraction and enrichment. The procedure opens new possibilities in medicinal diagnosis.

\section{Einleitung}

Seit langem werden die verschiedensten klinisch-chemischen Parameter des Liquors zur Diagnostik neurologischer und neurochirurgischer Krankheitsbilder herangezogen. Bei der Bestimmung von Kohlenhydraten in Körperflüssigkeiten hat man sich bisher auf wenige Zucker wie Glucose, Galaktose und Fructose beschränkt. Die Entwicklung eines automatischen Zuckeranalysators gibt dem klinischen Chemiker ein Werkzeug in die Hand, Zucker in Körperflüssigkeiten qualitativ und quantitativ routinemäßig in Nanomolmengen zu bestimmen. Am Beispiel der Untersuchung von Liquores von 24 Patien: ten sollen erste Anhaltspunkte über dạs Auftreten bishẹ im Liquor zum Teil unbekannter Zucker gewonnen werden.

\section{Methodik}

\section{Problematik der Zucker trennungen}

Gängige Methoden in der klinischen Chemie zur qualitativen Kohlenhydratbestimmung normaler und pathologischer Proben von Körperflitssigkeiten wie z. B. Dünnschichtchromatographie oder enzymatische Methóden $(1,2)$ genügen häufig nicht zur Untersuchung komplexer Kohlenhydratstoff wechselvorgänge.
Mit einem von uns in Zusammenarbeit mit der Fa. Biotronik GmbH., Frankfurt, entwickelten Zuckeranalysator (3-6) können zum Beispiel erstmals über 10 der in der klinischen Diagnostik am häufigsten auftretenden Kohlenhydrate in weniger als 30 Minuten aufgetrennt und nachgewiesen werden. Das Funktionsprinzip dieses Gerätes beruht auf dem von Khym \& Zill (7) zuerst beschriebenen und später von Kesler (8) vervollkommneten flüssigkeitschromatographischen Trennverfahren von Zucker-Borat-Komplexen an Ionenaustauschern mittels borathaltiger Elutionslösungen. Die Nachweisgrenze beträgt 0,05 bis $0,2 \mu \mathrm{g}$ je Komponente bei höchster Empfindlichkeit. Da zur photometrischen Detektion eine spezifische Anfärbereaktion (Orcin-Schwefelsäure (9)) durchgefuhrt wird, ist die Probenaufarbeitung einfach und problemlos: Nur hochmolekulare $\mathrm{Be}-$ standteile der Proben müssen dürch Ultramembranfiltration entfernt werden, um eine Verschmutzung des Trennsystems zu vermeiden.

Funktion und apparative Beschreibung des Zuckeranaly sators

In Abbildung 1 ist das Blockschema des Zuckeranalysators dargestellt. Gradientenkammer und Boratpuffervorratsbehälter sind über elektrisch gesteuerte Ventile mit einer Mikrodosierpumpe der Fa. Milton-Roy-Dosapro verbunden. Die Elutionslösungen werden druckseits mit einer Fördergeschwindigkeit von $1,3 \mathrm{ml} / \mathrm{min}$ über eine Vorwaschsäule (Dowex $1 \times 4$ ) auf die auf $60^{\circ} \mathrm{C}$ termostatisierte Trennsäule von nur $0,4 \times 8 \mathrm{~cm}$ geleitet. In der Säule befindet siç ein zu $4 \%$ quervernetzter, stark basischer, sphärischer Ionenaustauscher (DA-X 4F) einer Korngöße von $11 \pm 1 \mu \mathrm{m}$ der Fa. Dưrrum Chemical Corporation, Palo Alto, USA. 


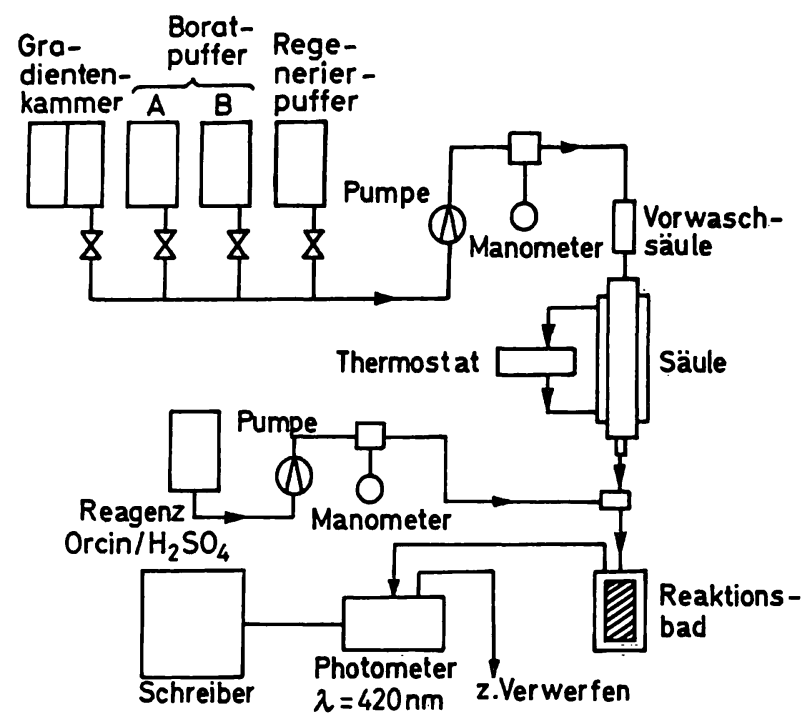

Abb. 1. Flußschaltbild des Zuckeranalysators.

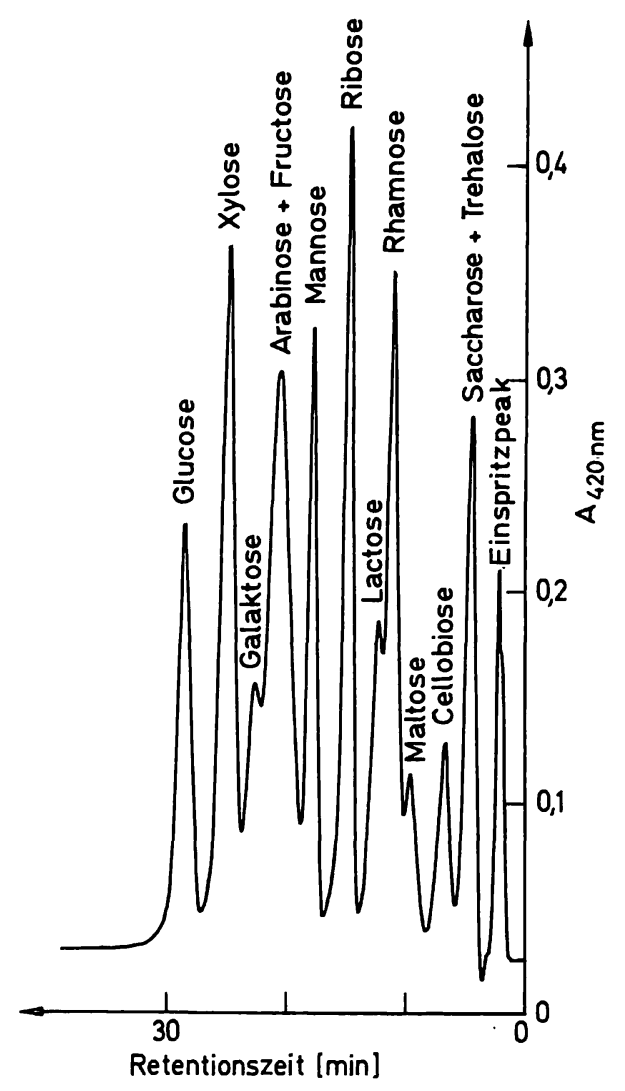

Abb. 2. Trennung eines Testgemisches.

Boratpuffer A: 0,1 mol $/ 1, \mathrm{pH} \mathrm{8,0;}$ Boratpuffer B: $0,4 \mathrm{~mol} / \mathrm{l}, \mathrm{pH} 9,2 ;$ Pufferwechsel: direkt nach Probenaufgabe; Säule: $0,4 \times 8 \mathrm{~cm}$; Förderrate $1,3 \mathrm{ml} / \mathrm{min}$; Säulentemperatur: $60^{\circ} \mathrm{C}$; Säulenrückdruck: $50-60$ bar; Schreiberempfindlichkeit: $A=0-0,5$; aufgetragene Menge: $60 \mathrm{nmol} / \mathrm{Mono}-$ und $30 \mathrm{nmol} /$ Disaccharid $(\hat{=} 9,0-10,8 \mu \mathrm{g})$

Das von der Säule kommende Eluat wird mit dem Reagens, $1 \mathrm{~g} / 1$ Orcin in konzentrierter Schwefelsäure, das mittels einer korrosionsbeständigen Mikrodosierpumpe gefördert wird, gemischt und der Farbstoff in einer Reaktionsspirale bei $100^{\circ} \mathrm{C}$ entwickelt. Die photometrische Bestimmung erfolgt bei $420 \mathrm{~nm}$ mit einem Filterphotometer mit variabel einstellbaren Absorptionsbereichen (LC 6620) der Fa. Biotronik, Frankfurt.
Abbildung 2 zeigt ein Diagramm eines Testgemisches mit 14 Komponenten. Es wird eine Stufenelution mit 2 Boratpuffern angewandt, wie sie für die hier diskutierten Untersuchungen von Liquor eingesetzt wird.

\section{Auswertung der Diagramme}

Die Zuordnung der einželnen Zucker erfolgt über die Retentions-Zeiten, die durch Kalibrierungsläufe ermittelt werden. Der Variationskoeffizient der Retentionszeiten ist für früher eluierte Zucker größer (3-5\%), als für später eluierte (2-3\%). Die quantitative Auswertung erfolgt über die Flächenberechnung (im allgemeinen nach der Formel: Höhe des Peaks multipliziert mit seiner Breite in halber Höhe) und der Multiplikation mit dem zuvor aus einem Kalibrierungslauf errechneten molaren Anfärbefaktor. Der Variationskoeffizient beträgt hierfür 2-3\%.

\section{Probenaufarbeitung}

Von 0,5 bis $1 \mathrm{ml}$ der zu untersuchenden Probèn werden in einer Micro-Ultramembranfiltrationszelle über ein Diaflo Ultrafilter mit einer Ausschlußgrenze von 1000 Dalton (UM 2) der Fa. Amicon Corporation unter Stickstoff (2 atm) hochmolekulare Komponenten abgetrennt. Von den Filtraten werden 20$100 \mu l$ ohne weitere Vorbehandlung direkt auf die Säule aufgetragen.

\section{Klinisches Untersuchung smaterial}

26 Lumballiquores und eine Serumprobe werden mit dem oben beschriebenen Analysator unter sucht. 4 charakteristische Zuckerchromatogramme von Liquor cerebrospinalis zeigen die Abbildungen 3-6.

18 Liquores werden bakteriologisch auf fölgenden Medien geprïft: Leberboullion nach Tarozzi, Blutagar, Schokoladenagar und Fuchsin-Lactoseagar nach Endo. Innerhalb von 24 Stunden werden die Proben zentrifugiert. Das Sediment wird verimpft und der Überstand bei $-40^{\circ} \mathrm{C}$ eingeforen. Die Zeit von der Probengewinnung bis zum Einfrieren beträgt zwischen null und sieben Tagen, im Mittel 1,8 Tage. Sechs Patienten leiden an eitriger, drei an Mumpsmeningitis. Zwei Patienten war kurz zuvor ein intracranielles Meningeom entfernt worden. Die Diagnosen sind in Tabelle 1 aufgefuihrt.

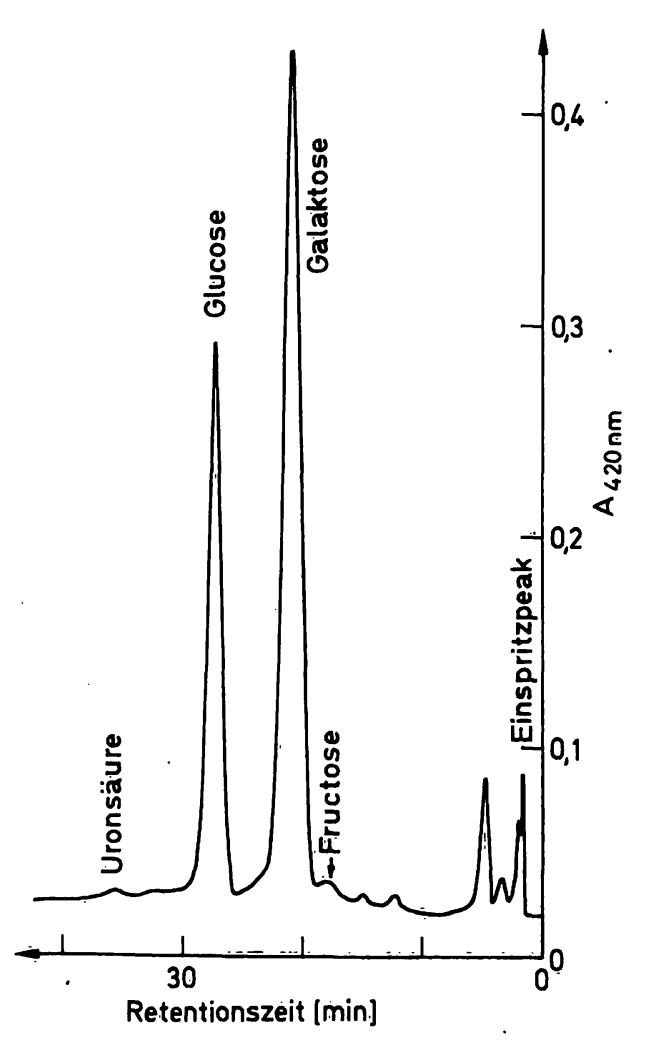

Abb. 3. Chromatogramm von 20 ul Lięuor, Patient 20, Versuchsbedingungen: siehe Abbildung, 2 . 


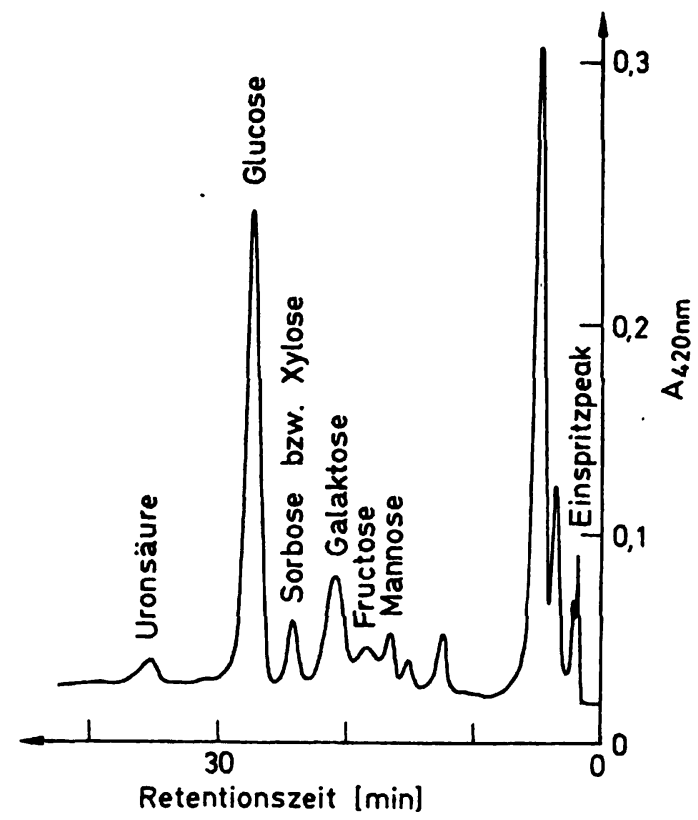

Abb. 4. Chromatogramm von $100 \mu l$ Liquor, Patient 21, Versuchsbedingungen: siehe Abbildung 2.

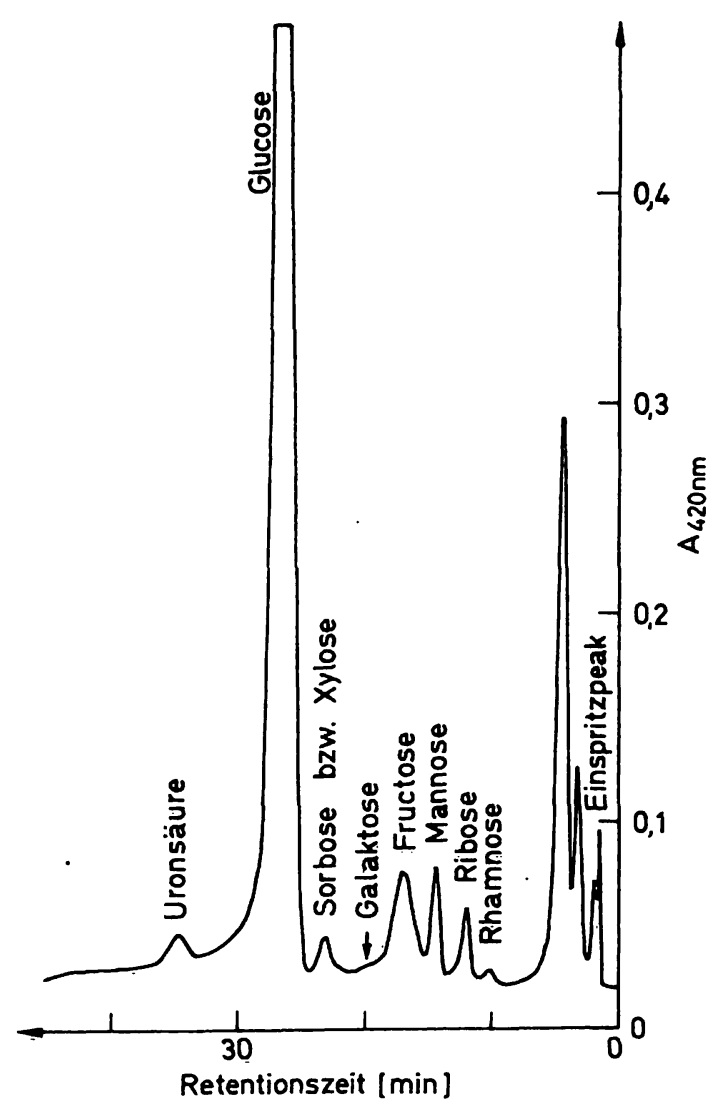

Abb. 6. Chromatogramm von $100 \mu$ l Liquor, Patient 22 (b), Versuchsbedingungen: siehe Abbildung 2.

\section{Ergebnisse und Diskussion}

Von den 18 bakteriolologịsch untersuchten Proben sing și 12 steril. Folgende Erreger werden isoliert: $H$. influenzae, Keime aus der Coli-Gruppe, N. meningitidis, Enterokok: ken $(2 \times)$, Diplococcus pneumoniae.

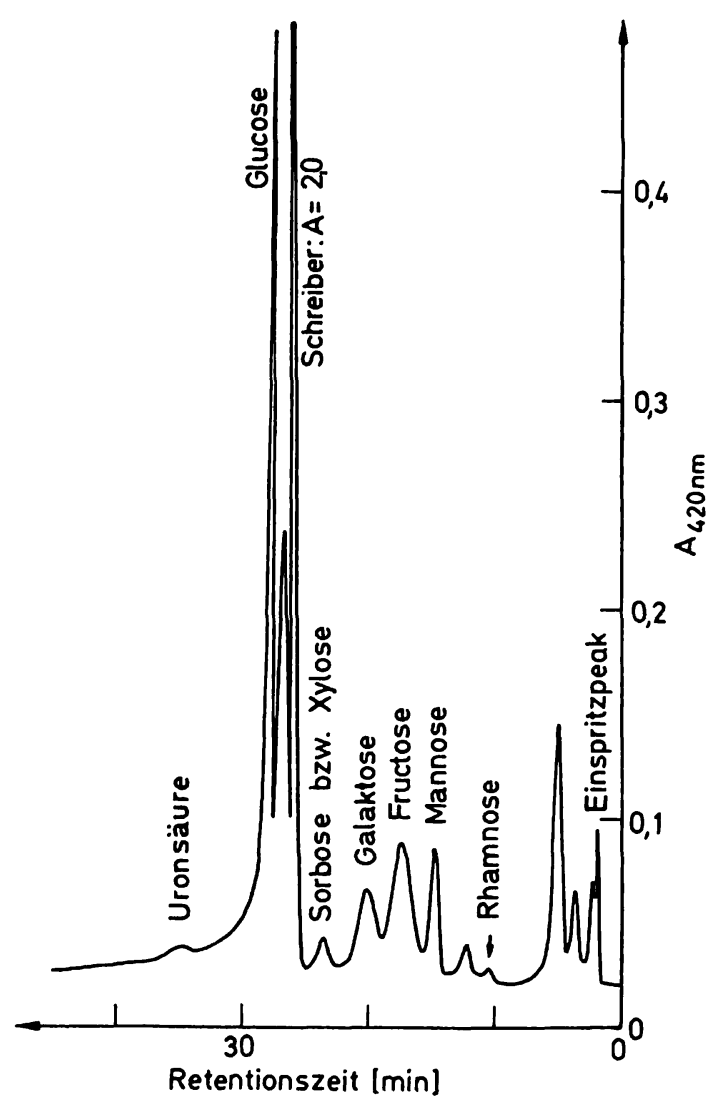

Abb. 5. Chromatogramm von $100 \mu$ l Liquor, Patient 22 (a), Versuchsbedingungen: siehe Abbildung 2.

Chromatographisch werden Substanzen mit den Retentionszeiten folgender Kohlenhydrate gefunden: Rhamnose, Ribose, Mannose, Fructose, Galaktose, Sorbose bzw. Xylose und Glucose. Die Konzentrationen sind in Tabelle 1 angegeben.

Neben Glucose und Fructose, die in jedem Liquor nachweisbar sind, findet man fast regelmäßig auch Ribose und Mannose. In je 6 Fällen treten Rhamnose und Galaktose auf. Die Fructosekonzentrationen liegen alle in derselben Größenordnung, im Mittel bei $0,21 \mathrm{mmol} / \mathrm{l}$. Mannose und Ribose liegen im Mittel bei 0,022 und $0,026 \mathrm{mmol} / \mathrm{l}$.

Interessant ist das Verhalten der Galaktose. Sie tritt in sechs Fällen auf, und zwar bei Meningeosis leucaemica, Mumpsmeningitis und Pneumokokkenmeningitis mit Werten zwischen 0,008 und $0,03 \mathrm{mmol} / \mathrm{l}$. Bei Patient 20 werden 10 Tage nach der:subtotalen Resektion eines Meningeoms im linken Stirnschläfenlappen 7,5 mmol/1 Galaktose und bei Fall 22 nach länger zurückliegender Meningeomoperation 0,13 mmol/l Galaktose nachgewiesen.

Bei der beschriebenen Methode sind mit Ausnahme der Uronsäuren nur Neutralzucker nachweisbar und wir haben hier erstmalig sechs verschiedene Zucker im Liquor cerebrospinalis feststellen können. In der uns bekannten Literatur wird über das Auftreten von Glucose, Glucosamin, Fructose und Inosit berichtet (10-13). In l. c. 


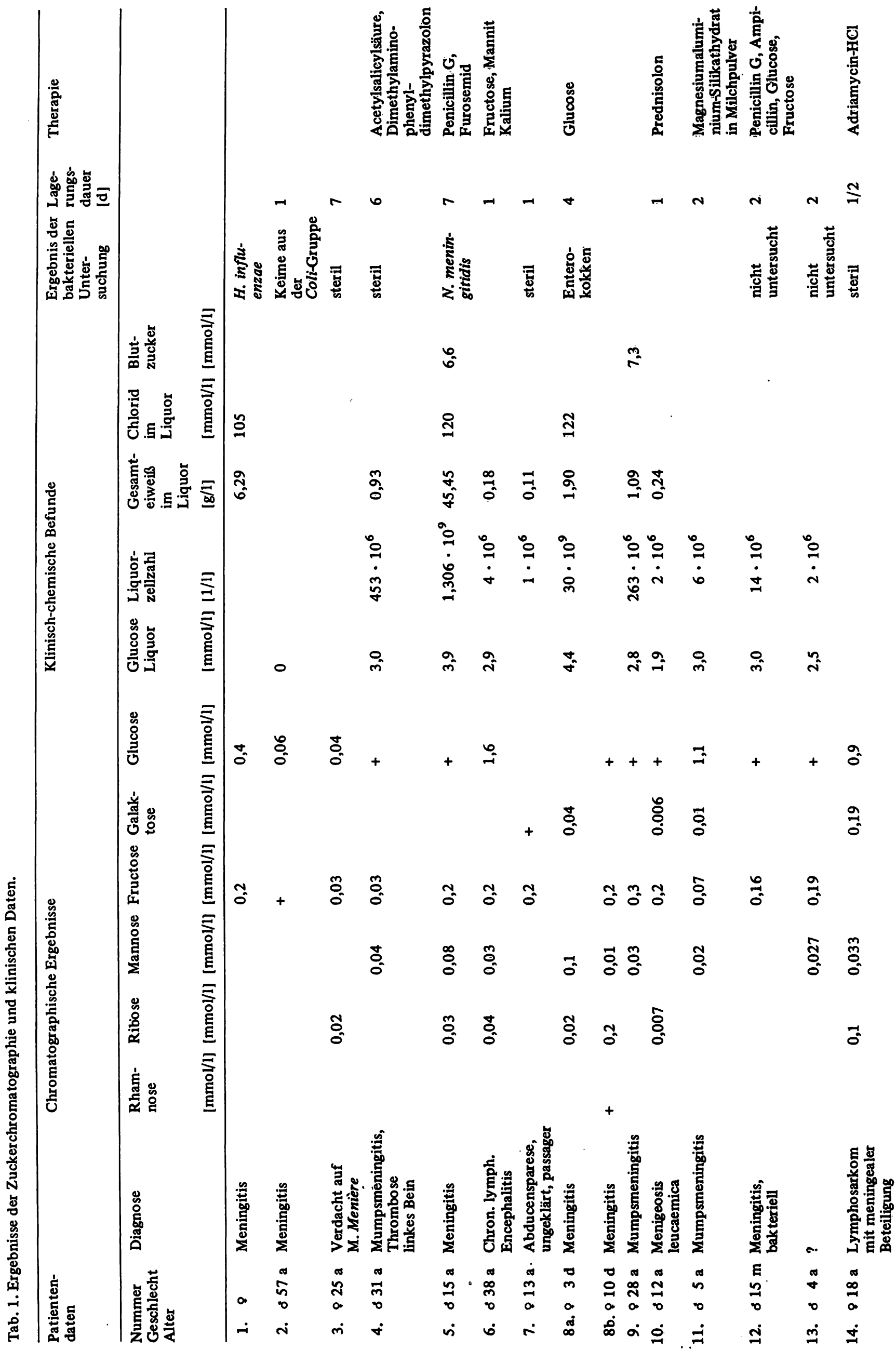



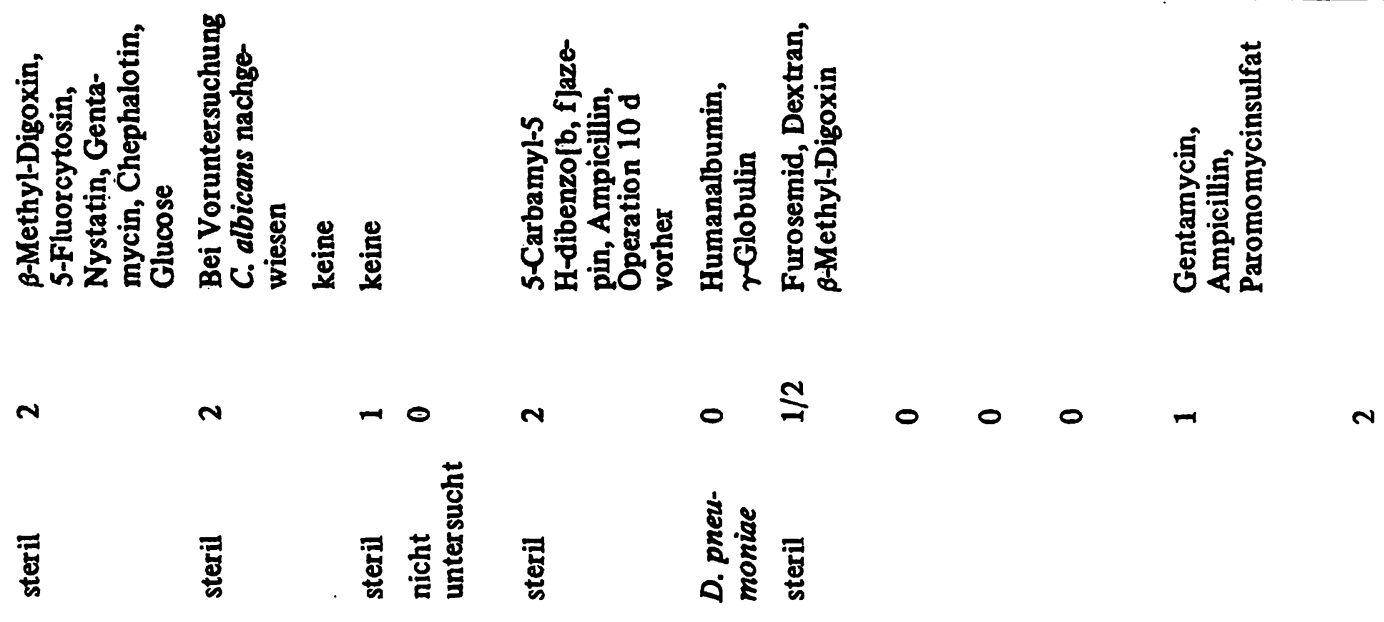

$\cong$

\begin{tabular}{|c|c|c|}
\hline & $\stackrel{\circ}{-}$ & : \\
\hline & 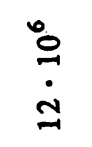 & $\stackrel{\circ}{\dddot{m}}$ \\
\hline & $\underset{\Im}{\tilde{I}}$ & \\
\hline
\end{tabular}

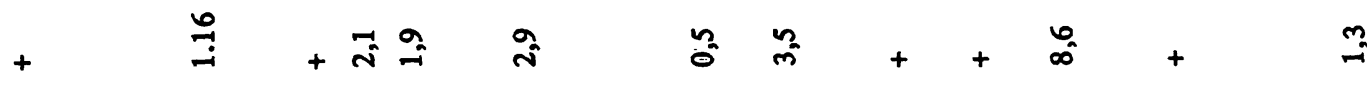

¿ำ

กู่ สูู

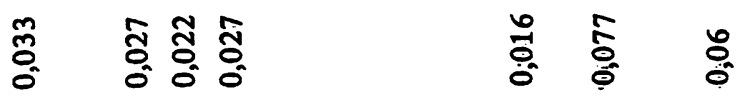

$\because \quad: \quad: 0$

항

क्ष

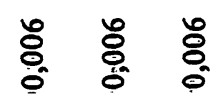
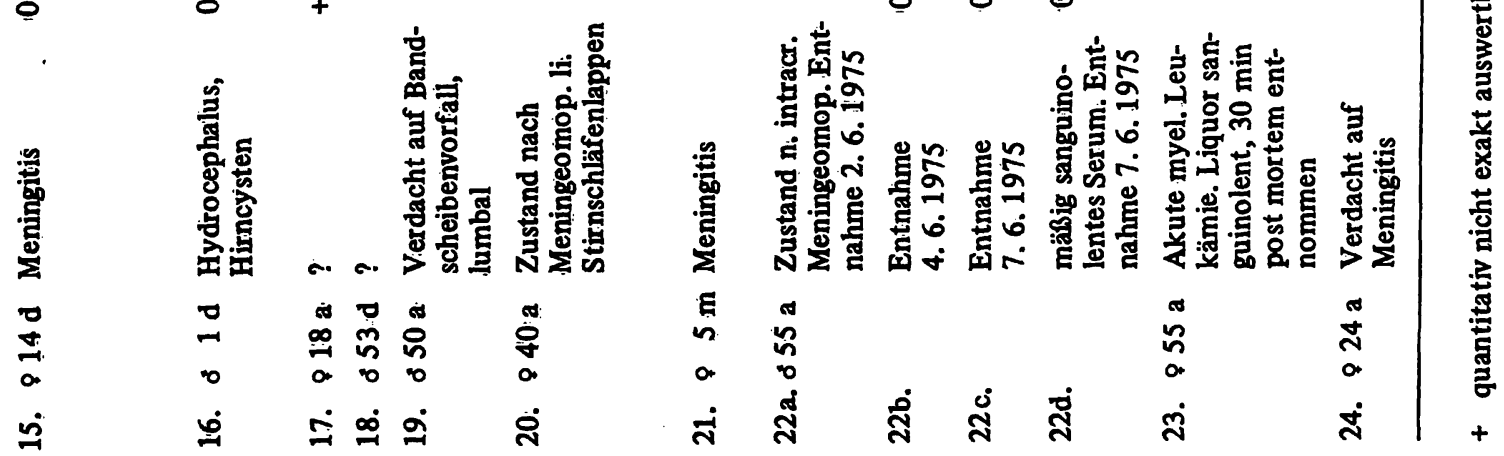
(7) wird über mittlere Fructosekonzentrationen im Lumballiquor von Erwachsenen von $0,18 \mathrm{mmol} / 1$ (0,13$0,23 \mathrm{mmol} / \mathrm{l})$ berichtet. Wir finden im Mittel $0,21 \mathrm{mmol} / \mathrm{l}$. Bemerkenswert sind die relativ geringen Schwankungen der Fructosekonzentration auch in Fällen erheblicher bakterieller Besiedelung, wie bei Patient 8, bei welchem die Glucosekonzentration auf $0,04 \mathrm{mmol} / 1$ herabgesunken ist. Niedrig liegen auch die Glucosekonzentrationen der Patienten 1, 2, 3 und 21. Bis auf Patient 3 handelt es sich um bakterielle Meningitiden. Es ist anzunehmen, daß die niederen Glucosespiegel durch bakteriellen Abbau entstehen. Bei Patient 2 konnte auch klinisch-chemisch wenige Stunden nach der Lumbalpunktion keine Glucose mehr nachgewiesen werden. Aus dem Nachweis von Ribose und Mannose können bis jetzt noch keine Schlüsse gezogen werden. Interessant ist, daß bei Patient 20 eine Galactosekonzentration von $7,5 \mathrm{mmol} / \mathrm{l}$ gefunden wird. Es handelt sich um einen 10 Tage zuvor an einem Meningeom operierten Patienten (linker Stirnschläfenlappen). Bei einem weiteren Meningeompatienten, 22, wird ebenfalls Galaktose, allerdings nur zu $0,13 \mathrm{mmol} / \mathrm{l}$ nachgewiesen. Die Operation liegt dabei einige Tage zurück. Vermutlich

\section{Literatur}

1. Curtius, H. C. \& Roth, M. (1974), „Clinical Biochemistry“ Walter de Gruyter, Berlin, New York.

2. Bergmeyer, H. U. (1974), ,Methoden der enzymatischen Analyse", Verlag Chemie, Weinheim.

3. Bauer, H. (1975), „Dissertation der Universität Tübingen“.

4. Voelter, W. \& Bauer, H. (1973), G-I-T 17, 846.

5. Voelter, W. \& Bauer, H. (1975), Clin. Chem. 21, 1882.

6. Bauer, H. \& Voelter, W. (1976), „Chromatographia“ 9, 433.

7. Khym, I. J. \& Zill, L. P. (1952), J. Am. Chem. Soc. 74, 2090.

8. Kesler, R. B. (1967), Analyt. Chem. 39, 1416. stammint die Galaktose aus Cerebrosiden des Gehirns. Dạ Aḅbạauprodukte der Gehirnsubstanz im Liquor auftauchen können (14) wäre unter Umständen eine diesbezügliche Diagnostik mittels Galaktose denkbar. Die Patienten 10, 11 und 25 lassen sich evtl. in diesem Sinne deutên. Bei Patient 10 handelt es sich möglicherweise um eine Störung der Blut-Liquorschranke.

Weitere Untersuchungen müßten der Frage nachgehen, ob mit Hilfe der Monosaccharide geringgradige Schädigungen der Blut-Liquorschranke erfaßbar sind. Die Fragen, die sich mit der Abhängigkeit der Zuckerkonzentration von der Höhe der Entnahmestelle oder von der Therapie ergeben, sind noch völlig offen. Wir meinen dennoch, eine Anregung für weitere Untersuchungen gegeben zu haben.

\section{Danksagung}

Wir danken den Herren Prof. Dr. R.-E. Bader (Tübingen), Privatdozent Dr. G. Rettenmaier (Böblingen) und Dr. W. Elies (Tübingen) für die UUberlassung von Liquorproben und klinischen Daten.

9. Pigman, W. \& Horton, D. (1970), „The Carbohydrates“, Academic Press, New York and London.

10. Papadopoulos, A. \& Hess, B. (1960), Arch. Biochem. Biophysics $88,167$.

11. Schmidt, R. M. (1968), „Der Liquor Cerebrospinalis“, Verlag VEB Volk und Gesundheit, Berlin.

12. Tourtellote, W. W., in ,Pathology of the Nervous System", Minckler, E., ed. S. 441, Mc Graw Hill, New York.

13. Gilland, O. (1971), Prog. Neurol. Psychiatry 26, 329-61.

14. Kanig, D. (1973), ,Einführung in die allgemeine und klinische Neurochemie", Gustav Fischer-Verlag, Stuttgart.

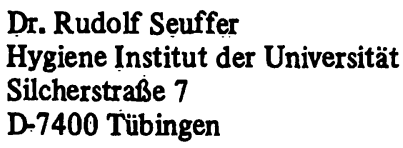

Prof. Dr. Wolfgang Voelter und Dr. Hermann Bauer Inștitut für Organische Chemie der Universität Auf der Morgenstelle D-7400 Tübingen 